\title{
Specificity of back muscle response to submaximal fatiguing contractions
}

\author{
Nils Fallentin ${ }^{\mathrm{a},},{ }^{*}$ Rammohan Maikala ${ }^{\mathrm{a},} \mathrm{Jacob}$ Banks $^{\mathrm{a}}$, Niall Obrien $^{\mathrm{a}}$ and Amanda Rivard ${ }^{\mathrm{a}}$ \\ ${ }^{a}$ Liberty Mutual Research Institute for Safety, 71 Frankland Road, Hopkinton, MA 01748, USA
}

\begin{abstract}
A fatigue-related decrease in quality and accuracy of the proprioceptive feedback from muscle spindles in the back (e.g. stretch reflexes) may lead to a deterioration of spinal stability, thereby making the spine more vulnerable to external perturbations. Endurance time, tissue oxygenation, reflex latency and blood pressure response were studied in twenty subjects during a submaximal endurance test of the back extensors $(20 \% \mathrm{MVC})$ performed as either a position task (supporting a weight stack while maintaining trunk position) or a force task (exerting a force against a dynamometer). Mean endurance time was $8.91( \pm 2.79) \mathrm{min}$ for the force task and $10.86( \pm 6.93) \mathrm{min}$ for the position task. This result is in striking contrast to what has previously been reported from limb muscles, and suggests that back muscles are especially well suited for postural tasks. An increased reflex latency found after the position task indicates that the disadvantage of the prolonged endurance time is a deterioration of the afferent input from the muscle spindles that may potentially hamper the protection of the spine
\end{abstract}

Keywords: Back muscle fatigue, reflex responses, cumulative trauma disorders, heavy lifting

\section{Introduction}

A fatigue-related decrease in quality and accuracy of the proprioceptive feedback from muscle spindles in the back (e.g. stretch reflexes) to the central nervous system may lead to a deterioration of the neuromuscular control of spinal stability, thereby making the spine more vulnerable to external perturbations. Enoka et al. [1] studied this neuromuscular interaction in limb muscles by comparing the demands of two fatigue tasks requiring similar net muscle torques, but imposing different demands on the nervous system. The found that a position tasks (i.e., supporting an inertial load while maintaining the limb in the same position) was experienced as more demanding and exhibiting a markedly shorter endurance time than exerting a comparable muscle torque against a rigid restraint ( a force task). Enoka and co-workers attributed such difference in fatigability to greater stretch reflex responsiveness in the position task leading to a more rapid recruitment of the motor neuron pool and a briefer time to task failure. However, the effect of task characteristics on endurance time and neuromuscular control has never been studied, in the back muscles and it was the aim of the present study to elucidate similarities and dissimilarities in back and limb muscle responses to force and position tasks.

Additionally, the study of fatigue characteristics of the low back muscles is essential for understanding the injury mechanisms in occupational low back pain. Work- related low back pain associated with overexertion may appear as a single event e.g. lifting a heavy burden, but in most cases, this event is merely the tip of the iceberg. The single lift represents the final outcome of a cumulative trauma pathway that may weakened the load tolerance of the back and create the prerequisite for an injury [2]. In this injury pathway fatigue of the low back muscles is the central component. The afferent input from the back muscles is decisive for maintaining a stable and well protected spine and a fatigue related decrease in the quality of this input will render the back open to injury.

\footnotetext{
${ }^{*}$ Corresponding author. E-mail: Nils.Fallentin@LibertyMutual.com
} 


\section{Methods}

\subsection{Subjects}

Twenty healthy men (mean age $25 \pm 4$ years) volunteered for the study. Mean body height was $175.6 \pm 8.6 \mathrm{~cm}$ and mean body weight $80.6 \pm 17.6 \mathrm{~kg}$. All subjects were screened for any neurological, muscular or cardiovascular disorders and written informed consent was obtained prior to the study. The Study was approved by the Internal Review Board at Liberty Mutual Research Institute.

\subsection{Procedure}

Each subject participated in three sessions. Maximal voluntary contraction (MVC) of the back extensor muscles was determined on an initial visit to the lab using a standardized test procedure [3]. At two subsequent sessions a submaximal endurance test of the back extensors (20\% MVC) was performed as either a position task (supporting a weight stack while maintaining an upright trunk position) or a force task (exerting a comparable force against a dynamometer.

In the force tasks, subjects were asked to match the target force displayed on a monitor and verbally encouraged to keep the force for as long as possible. Termination of the task was defined as the time when the force could no longer be maintained and this time was recorded as the endurance time. In the position tasks, subjects were asked to keep the posture of the torso constant for as long as possible and verbally encouraged to do so. Visual feedback on deviations in posture was provided and the time when the required posture could no longer be maintained was recorded as the endurance time for the position task,

The sequence of the two types of contraction was balanced and several days of recovery were allowed between trials.

\subsection{Experimental set up}

The experimental set up is shown in Fig. 1.

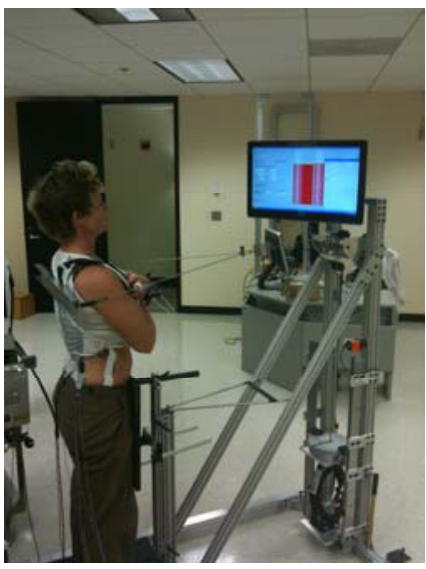

Fig.1. Experimental set up

In the force task the wire was attached to the floor and passed a reel before being fastened to a rigid bar across the back by mean of a harness. The horizontal force exerted by the subject during an attempted trunk extension was measured by a strain gauge dynamometer inserted between the floor attachment and the reel. Subjects were standing erect facing the dynamometer with the pelvis supported. In the position task a load was attached to the wire running over the reel, thus transmitting the inertial (gravitational) force of the load as a horizontal force applied to the trunk. The position of the trunk was monitored by a potentiometer mounted on the reel, while the subject was fixed at the hip to restrain any movements to the isolated trunk.

\subsection{Measurements}

Blood pressure, tissue oxygenation and back muscle electromyography (EMG) were measured continuously during the experiments while reflex responses were assessed before and immediately after the termination of the task.

\subsubsection{Blood pressure}

Blood pressure was monitored with an automatic beat-by-beat blood pressure monitor (Finapres 2300, Ohmeda) using the Penaz principle. The measuring cuff was placed on the middle finger and any deviations in the blood pressure recording due to the removal of the hand from heart level were automatically corrected. 


\subsubsection{Tissue oxygenation and blood volume}

Tissue oxygenation and total hemoglobin $(\mathrm{Hb})$ concentration in the erector spinae muscle were monitored using a continuous wave near-infrared spectroscopy system (NIRO-300, Hamamatsu Photonics, Japan). By using a spatially resolved technique the system provides a Tissue Oxygenation Index (TOI) defined as the ratio of $\mathrm{HbO}_{2} /(\mathrm{HbO} 2+$ $\mathrm{HHb}$ ) and is expressed in percentage. In addition the total $\mathrm{Hb}$ content in the tissue was obtained from the system and used as an indicator for changes in muscle blood volume. The optical sensors were placed on the left erector spinae muscle of the subject at a level corresponding to L3. The detector in the optical sensor was separated from the emitter by $4 \mathrm{~cm}$.

\subsection{3. $E M G$}

Surface EMG was recorded bilaterally from the erector spinae muscles at a level corresponding to L1, from the right erector spinae muscle at 13 (opposite the NIRS recording probe) and from the right and left multifidus (Motion Lab Systems). EMG data not reported in this paper.

\subsubsection{Reflex responses}

Fatigue induced changes in reflex responsiveness were assessed by direct tapping of the erector spinae muscles. The technique for measuring reflex response has been described in detail in Skotte el al [3]. Briefly, a reflex response is elicited using an electromechanical tapping system consisting of a prodder and a position sensor fixed to the armature of a solenoid. The taps are made on a cap $1 \mathrm{~cm}$ in diameter attached to the skin of the subjects at L3 level. The cap and the end of the prodder make an electrical contact for detecting tap onset, while the position sensor measures the position of the prodder i.e. the depth of the tap. To characterize the reflex response, 25 taps with a duration of 3-4 ms and a depth of 5 $\mathrm{mm}$ were applied to the right erector spinae muscle of the subjects before and immediately after task termination, and sampled together with the associated EMG response. In the results section below, the mean reflex responsiveness - average of the 25 taps - is presented as latency (time from prodder contact to EMG onset) and mean-peak to peak amplitude of the M1 response (ratio of the peak-to-peak amplitude in relation to the background EMG activity). For this proceeding paper latency and amplitude values were determined using a customized software program.

\subsubsection{Statistics}

Results are presented as mean values \pm SD (in figures only man values) Physiological responses during the two tasks were compared using paired samples ttests. The level of significance was set at $\mathrm{P}<0.05$.

\section{Results}

\subsection{Time to task failure (endurance time)}

Mean endurance time was $8.91( \pm 2.79) \mathrm{min}$ for the force task and $10.86( \pm 6.93)$ min for the position task. The difference between tasks was not significant $(\mathrm{P}=0.159)$, but the majority of the subjects exhibited longer endurance time during the position task.

\subsection{Blood pressure response}

Mean arterial blood pressure (MAP) increased continuously from resting values of approximately $80 \mathrm{~mm} \mathrm{Hg}$ to end values of $95 \pm 15$ (position task) and $101 \pm 14 \mathrm{~mm} \mathrm{Hg}$ (force task), respectively. The observed difference in MAP at task failure was not significant $\mathrm{P}=0.163$.

The blood pressure response is shown in fig 2 .

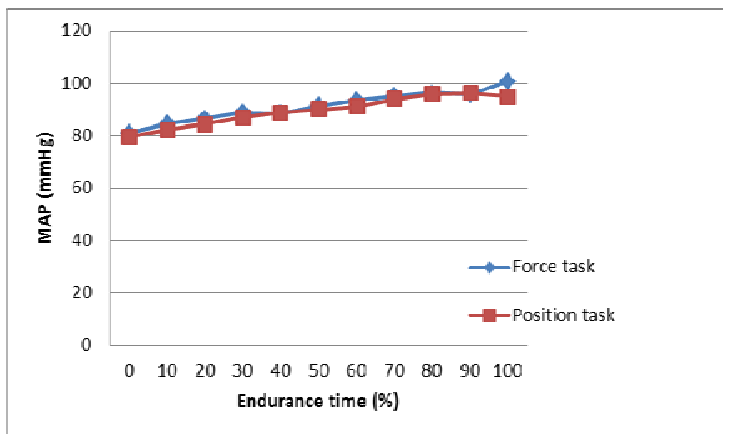

Fig.2. Mean arterial pressure (MAP) during the force and the position task. Values are normalized to percent endurance time $(\mathrm{n}=14$; only subjects with a complete data set were included).

\subsection{Tissue oxygenation}

Mean TOI values in the two experimental conditions are shown in fig. 3. When normalized to percent endurance time there is a modest decrease in tissue oxygenation during the course of both tasks. TOI values were reduced from initial values of $72.5 \pm 6.3 \%$ (position task) and $72.0 \pm 7.1 \%$ (force task) to $67.0 \pm .8 .0 \%$ and $67.0 \pm 8.8 \%$, respectively. 


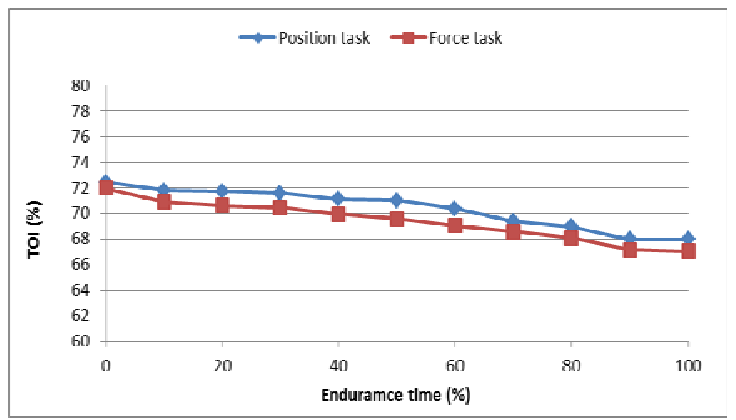

Fig.3 Tissue oxygenation index (TOI) during the force and the position task. Values are normalized to percent endurance time ( $\mathrm{n}=16$; only subjects with a complete data set were included).

The modest decrease in TOI was possible due to a similtaneous but opposite changes in tissue blood volume values increasing steadily during the experiment. (Fig. 4).

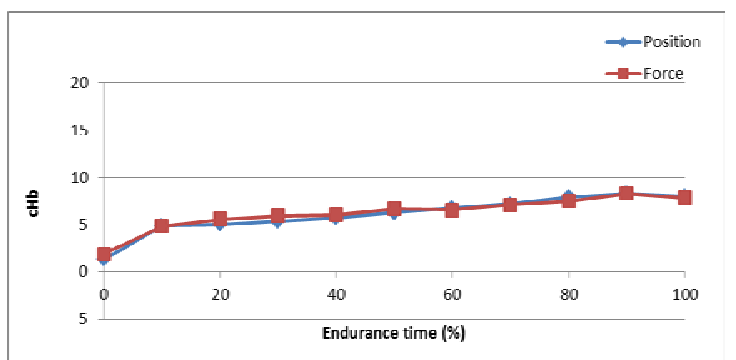

Fig. 4. Total hemoglobin content $(\mu \mathrm{M})$ in the tissue during the force and the position task Values are normalized to percent endurance time $(n=16$; only subjects with a complete data set were included).

\subsection{Reflex responsiveness}

Despite the relative moderate changes in tissue oxygenation the reflex responsiveness was affected at task termination. The mean reflex latency increased significantly in the position task from $9,84 \pm 5.40 \mathrm{~ms}$ pre experiment to $11.58 \pm 6.58 \mathrm{~ms}$ post experiment. The changes in reflex latency in the force tasks was only borderline significant $(\mathrm{p}=0.053)$ but the latency increased from $12.11 \pm 6.60 \mathrm{~ms}$ to $14.67 \pm 6.84 \mathrm{~ms}$.

The amplitude of the elicited reflex response decreased in the position task, but increased in the force task. Neither the decrease in amplitude values in the position task (from a ratio of $5.1 \pm 6.1$ to a ratio of $3.8 \pm 2.5$ ) nor the increase in the force tasks (from a ratio of $2.8 \pm 1.4$ to $3.5 \pm 2.8$ ) was, however, significant.

\section{Discussion}

The results demonstrated a marked difference between back and limb muscle responses to force and position tasks. The endurance time for the back muscles was not significantly different in the two tasks with most of the subjects having the longest endurance time in the position task - which is in striking contrast to what has been reported from most other muscles. In a review paper summarizing the results from a number of studies, Enoka found that in general the endurance time was around $40-50 \%$ shorter for position tasks [1]. The cardiovascular responses mimic this difference in endurance time. When Hunter at al compared the cardiovascular responses to a position and a force task with the elbow flexors, a more rapid increase in MAP and a higher end value was observed during the position task [4]. Again results markedly different from the present study. The time course of the blood pressure response when the two task were performed with the back muscles were almost identical (see fig 2) and the end MAP values were actually higher in the force experiments (difference not significant) i.e. a completely reversed pattern.

The main reason for looking at difference in task related fatigue is the potential insight that can be gained into neuromuscular control mechanisms and the role of muscle stretch reflexes and proprioceptive feed-back. In this context, the short endurance time in the potion tasks has been explained as increased stretch reflex sensitivity (due to gamma activation) required for maintaining a constant position with a compliant load. As a consequence of this strategy there is a more rapid recruitment of the motor neuron pool and a briefer time to task failure when supporting a compliant load.

This phenomenon does not occur in the back muscles and the varied response pattern suggests a different function of the muscle spindles in different muscle synergies. The reflex induced recruitment of motor units in the limb muscle is sub-optimal from an endurance point of view and it can be speculated that postural muscles are genetically better suited to maintain a constant posture against an external load (or gravitational forces). This idea gains some support from allometric analysis of the number of muscle spindles in human skeletal muscle showing that the greatest relative spindle abundance was found in the axial muscles i.e. the muscles around the vertebral column [5]. 
From a practical ergonomic standpoint the capacity of the back muscle to sustain a posture for a long time appears beneficial. The drawback, however, is that in the end, this prolonged endurance in a postural task causes a deterioration of the responsiveness and accuracy of the muscle spindle system. The increased reflex latency found after the posture task illustrates a deterioration of the afferents input from the muscle spindles that may potentially hamper the protection of the spine, As mentioned in the introduction, the ability of the muscular system to control spinal stability is to a large extent based on feedback provided from the back muscles, The proprioceptive information from muscle spindles is essential to maintain adequate timing and magnitude of muscle contraction that can efficiently protect the spine and ensure that kinematic disturbances can be controlled and attenuated.

The problem may be further aggravated by pronounced inter individual differences in response characteristics. The relatively high similarity between the force and potion task fact when looking at the average response pattern tends to obscure the fact that while some individual had markedly longer endurance time in the position task than in the force tasks others performed very poorly in the position task i.e. had a response more closely mimicking the limp response.

Fig $5 \mathrm{a}$ and $\mathrm{b}$ present TOI and MAP values from a subject with the best performance in the position task (endurance time $921 \mathrm{~s}$ vs., $583 \mathrm{~s}$ in the force task). Even at the point of task failure in the position task the subject exhibited an actual increase in tissue oxygenation and only a modest increase in blood pressure. The figure is not typical, but illustrates what is probably an optimal adaptation to a position task. The point to be made, however, is that at the other end of the spectrum there are "high risk" responders with the shortest endurance time in position task and possibly an increased vulnerability to sudden perturbations in connection with heavy lifting.

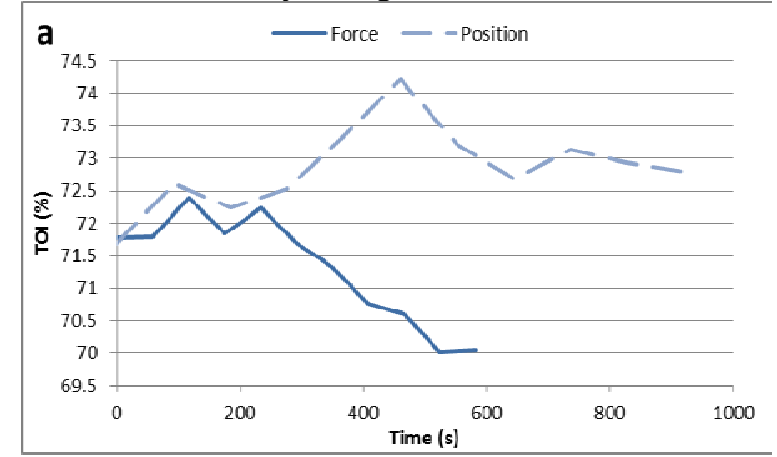

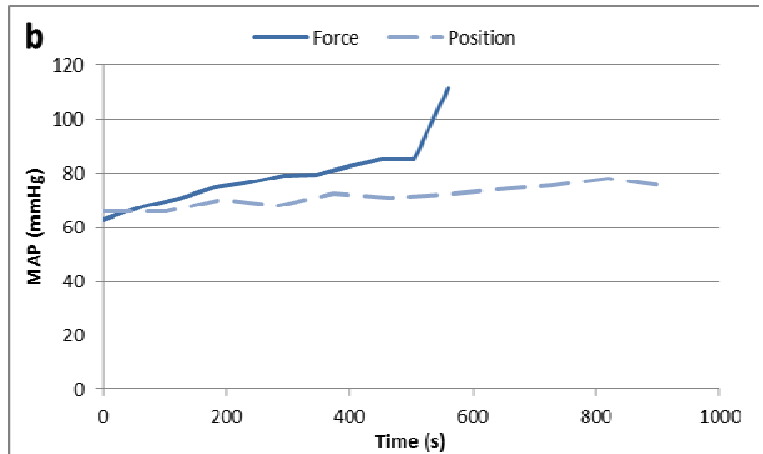

Fig.5 a and b. TOI and MAP values from a subject with the best performance in the position task (endurance time $921 \mathrm{~s}$ vs., 583 $\mathrm{s}$ in the force task)

A number of physical characteristics and training status may explain this marked difference in individual response patterns. One factor could be the ability to maintain adequate tissue oxygenation throughout the experiment. The time course of the mean TOI response shown in fig. 3 seem to indicate that the decline in tissue oxygenation is modest and predictive and, at any given point, mainly determined by the percentage of maximal endurance time.

However, when comparing the best and worst performer in the position task (fig.6) it is obvious that even when normalized to $100 \%$ endurance time the best performers are able to maintain a higher TOI throughout the task.

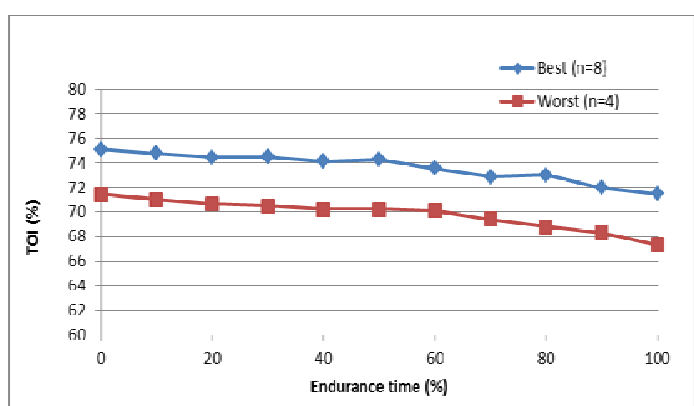

Fig. 6. Tissue oxygenation index for best $(n=8)$ and worst $(n=4)$ performers in the position task. Values are normalized to percent endurance time 
In conclusion, the present study demonstrated that although the back muscles are well suited for prolonged postural loading, sustained contractions ultimately lead to a deterioration of the load tolerance of the spine. The differences in individual response patters at the same time indicate that some individuals may experience a substantially higher risk for back injuries..

\section{References}

[1] R.M Enoka, S. Baudry, T. Rudroff, D. Farina, M. Klass, and J. Duchateau. Unraveling the neurophysiology of muscle fatigue. J Electromyogr Kinesiol 21 (2011), 208-19.

[2] S.M. McGill. The biomechanics of low back injury: implications on current practice in industry and the clinic. J Biomechanics 30 (1997), 465-75.

[3] J. Skotte N. Hjortskov, M. Essendrop, B. Schibye, and N. Fallentin. Short latency stretch reflex in human lumbar Para spinal muscles. J Neurosci Methods 145 (2005), 145-50.

[4] S.K. Hunter, D.L. Ryan, J.D. Ortega, and R.M. Enoka. Task differences with the same load torque alter the endurance time of submaximal fatiguing contractions in humans. J Neurophysiol 88 (2002), 3087-96.

[5] R.W. Banks. An allometric analysis of the number of muscle spindles in mammalian skeletal muscles. J Anat 208 (2006), 753-68. 